\title{
Method for the fuzzy control system synthesis for the analytical approximation characteristics of the typical nonlinear elements characteristics
}

\author{
$V$ Lubentsov $^{1, *}, E$ Shakhrai ${ }^{1}$ and $E$ Lubentsova $^{1}$ \\ ${ }^{1}$ Kuban state technological University, Krasnodar, Russia
}

\begin{abstract}
The solution of the problem concerning the control system synthesis with a fuzzy controller on the basis of typical nonlinear analytical approximation characteristics with continuous differentiable functions like sigmoid type has been considered. A method of synthesis which is based on the fact that the rulebase and membership function are formed by the way of comparing the control or corrective impacts of the fuzzy controller (prototype) or a controller with approximating control (CAC) has been suggested for problem solving. For the CAC construction the closest coincidence of graphically installed controllers' impacts are taken. This makes it possible to exclude subjective errors connecting with a possible non-representative ruleset for the rulebase forming. The result of the work is to obtain a new class of controllers based on the approximating continuous functions, which are simpler for constructing multi-mode control systems. The practical significance lies in the fact, that the tuning of the fuzzy controller based on the analytical continuous approximation of the typical nonlinear elements' characteristics comes down to two basic parameters: the coefficient that determines the curvature (slope) of the characteristic in the transit mode and the parameter that determines the control impact value in the static mode. The advantage of this smooth characteristic in relation to the piecewise linear approximation is that there is no need to determine the moments (points) of switching nonlinear characteristics during the system operation.
\end{abstract}

\section{Introduction}

The improvement in the quality of control was due to the difficulty of obtaining adequate mathematical models for many industrial processes. In such cases, traditional methods of synthesis of automatic control systems (ACS) based on simplified, inaccurate models do not always lead to the desired results. This determines the advisability of using methods that take into account the inaccuracy of models. These methods include methods for constructing control systems with fuzzy logic [1]. However, despite the advantages of fuzzy control systems (the design of fuzzy controllers does not require a mathematical description of the dynamics of a controlled object, and it is assumed that there is empirical knowledge about setting up controllers for the rulebase (RB) [2-5]), there are no universal

* Corresponding author: vf.lubentsov@yandex.ru 
methods for forming rulebases (RB) and membership functions (MF). The formation of rulebases is usually carried out on the basis of empirical knowledge about the control process, which does not exclude subjective errors caused by the human factor. Therefore, the development of a method for the synthesis of fuzzy control systems, including the choice of rulebases and membership functions, is an urgent task.

The most simple and common for control problems are triangular membership functions with the following linguistic terms [6, 7]: NL - negative large, PL - positive large, NM negative mean, AZ - zero, PM - positive mean, NS - negative small, PS - positive small.

The article [8] considers examples of a fuzzy controller with intersection in the input and (or) output triangular membership functions. These examples show that the type of characteristic of a fuzzy controller depends only on the rulebase and the membership functions of the input and output variables. The mismatch signal $\varepsilon_{k}$ and the rate of its change are used as input variables, and the control or corrective action $u_{k}$ at the $k$-th time moment $t_{k}(k=0,1,2)$ is used as the output variable. As a rule, input and output values of the variables are normalized in the interval [-1;1]. For example, for the stabilization mode, we take three linguistic terms: NS - negative small, AZ - zero, PS - positive small. The rulebase of a fuzzy controller with membership functions presented in Figures 1,a and 1,b will be as follows:

$$
\begin{aligned}
& \text { If } \varepsilon_{k}=\mathrm{NS} \text {, then } u_{k}=\mathrm{NS} ; \\
& \text { If } \varepsilon_{k}=\mathrm{AZ} \text {, then } u_{k}=\mathrm{AZ} ; \\
& \text { If } \varepsilon_{k}=\mathrm{PS} \text {, then } u_{k}=\mathrm{PS} .
\end{aligned}
$$

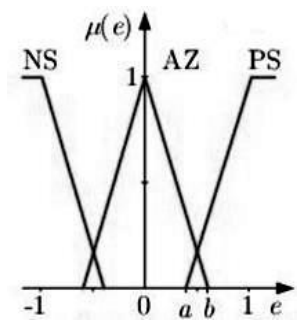

a)

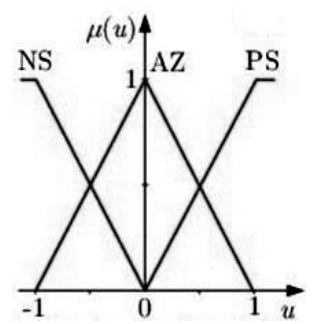

b)

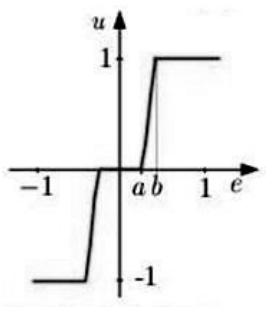

c)

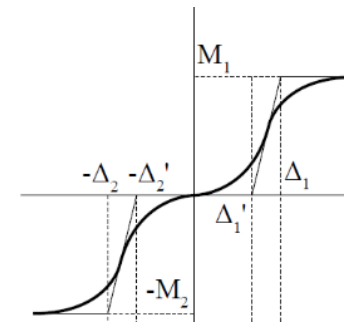

d)

Fig. 1. Membership functions of the error (a), control action (b), static characteristics of the fuzzy controller (c) and the CAC-controller (d), approximating the relay characteristic with the dead zone.

The membership function of the input variable $\varepsilon$ will have, for example, two intersections (overlaps) on the intervals $[-0.6,-0.4]$ and $[0.4,0.6]$, corresponding to the ranges of positive slopes of the curve. The static characteristic of the CAC-controller is approximately piecewise linear, clearly having three levels.

If the number of linguistic terms in the input and output membership functions is increased, then the characteristic remains similar, but with a large number of parts. The number of parts depends only on the number of linguistic terms, and the width of the parts depends on the degree of intersection. If there are no intersections in the input membership function, the characteristic of the controller with three states is obtained as shown in Figure 2. In this case, only one rule will be active, as a result of which three clear values appear at the output: $-1,0,1$. In this case, the static characteristic of the CAC-controller remains similar to the previous characteristic presented in Figure 1. 


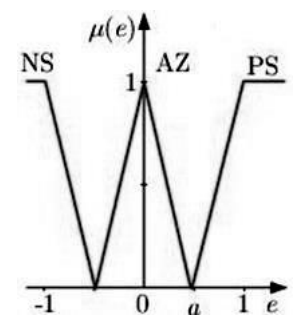

a)

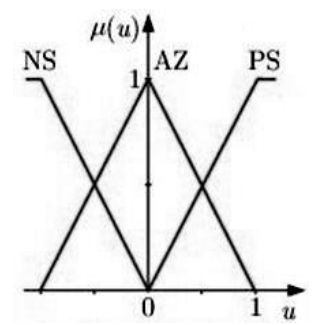

b)

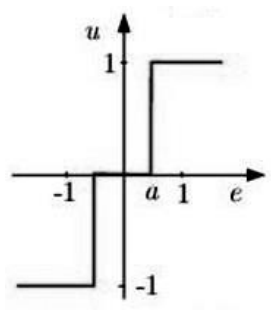

c)

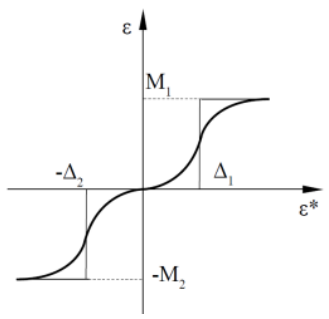

d)

Fig. 2. Membership functions of the error (a) without intersections in the input membership function, control action (b) of a fuzzy controller with complete intersection in the output membership function and static characteristics of the fuzzy controller (c) and the CAC-controller (d), approximating the relay characteristic with the dead zone.

Next, we will consider changes in the degree of intersection of the output membership function. Figure 3 presents the full intersection of the input and output membership functions.

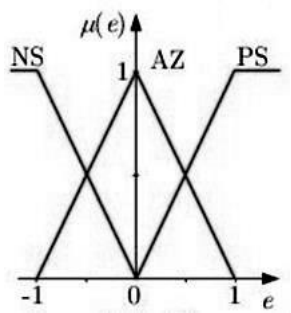

a)

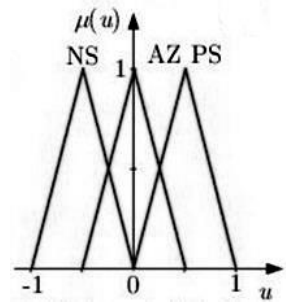

b)

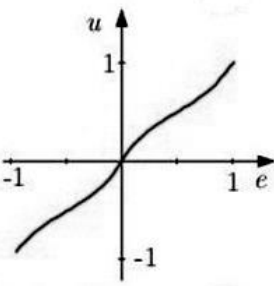

c)

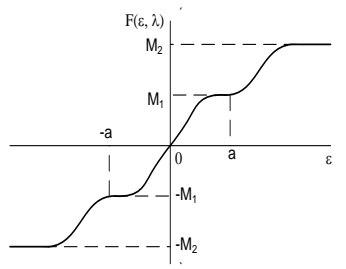

d)

Fig. 3. Membership functions of the error (a), control action (b) of a fuzzy controller with complete intersection in the input and output membership functions and static characteristics of the fuzzy controller (c) and the CAC-controller (d), approximating two relay characteristics without a zone and with the dead zone.

It can be expected that the level of intersection in the input membership functions has a strong effect on the statics of a fuzzy controller, namely, with the help of small intersections in the input membership functions, threshold (step) characteristics are formed, and with the help of large ones, the smoothness of the curve is achieved. Intersections in the output membership functions have a lesser effect on the controller characteristics. Figure 4 presents membership functions with full intersection at the input and no intersection at the output.

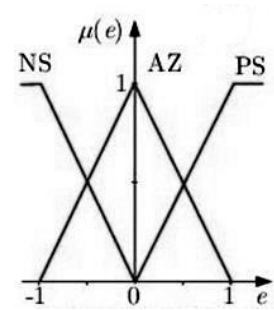

a)

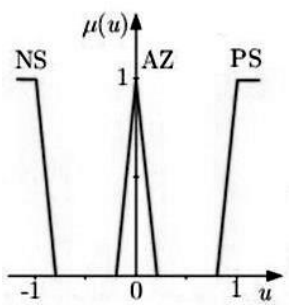

b)

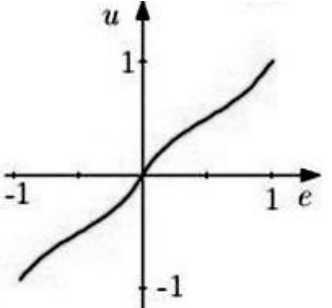

c)

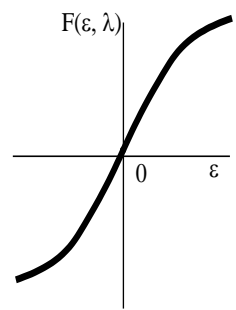

d)

Fig. 4. Membership functions of the error (a), control action (b) of a fuzzy controller with full intersection of the input membership function and without intersection of the output membership functions (b), static characteristics of the fuzzy controller (c) and CAC-controller (d). 
The size of each output membership function strongly affects the characteristic [7]. Figure 5 shows the case for a very small base of the membership function AZ (zero), which was created as an S-type characteristic with a large gain at the origin.

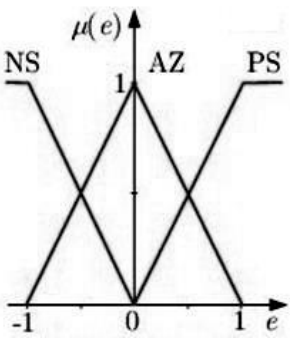

a)

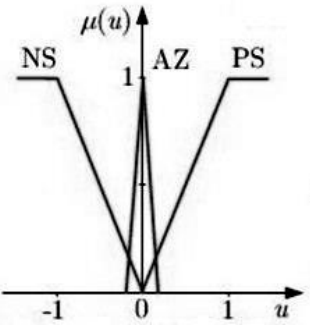

b)

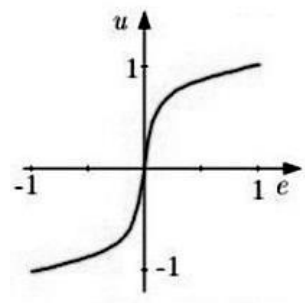

c)

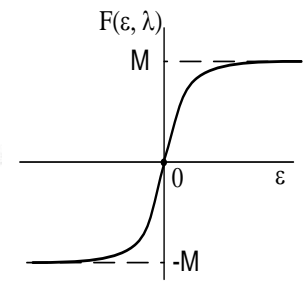

d)

Fig. 5. Effect on the characteristic (c) of a fuzzy controller with full intersection of the input membership functions (a) of the small base AZ (zero) in the output membership function (b) with the intersection.

The extension of the basis of the membership function AZ inverts (reverses) the S-curve with a small gain at the origin, as shown in Figure 6. Thus, the shape of the characteristic is highly dependent on the base of each output membership function.

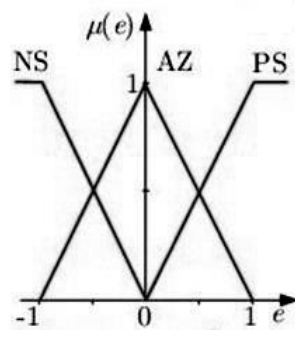

a)

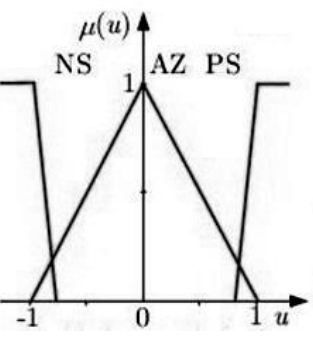

b)

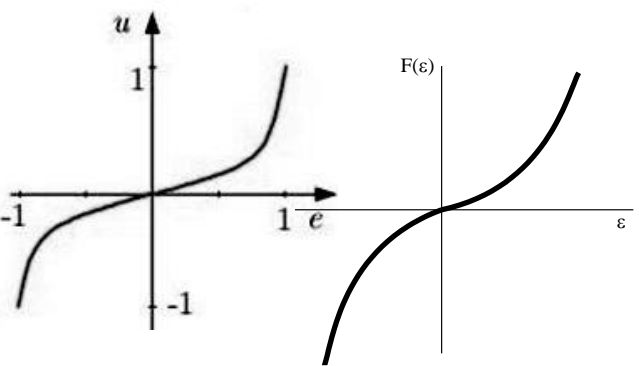

c) d)

Fig. 6. Effect on the characteristic (c) of a fuzzy controller with full intersection of the input membership functions (a) of the large base AZ (zero) in the output membership function (b).

The analysis of the presented graphs showed that the characteristic, and hence the quality of control, will depend on the applied membership function and the degree of overlap of the bases of the input membership functions in the output membership function. Since there are a large number of such options, the task of selecting them for specific implementations of fuzzy controllers is important. This confirms that there are no universal recommendations for selecting these intersections and the existing control algorithms are still far from perfect, and the controllers created on their basis need to be improved. Moreover, as shown in Figures 5 and 6, an equivalent output characteristic of the CAC-controller can be selected for each output characteristic of the fuzzy controller (FC). Taking into account the above, this work proposes an approach to the synthesis of a fuzzy controller of an automatic control system, taking into account the influence on the control characteristic of the parameters of the membership function. At its core, it uses the equivalence of graphical control dependencies $U(\varepsilon)$ on the mismatch signal $\varepsilon$ obtained by approximating nonlinear discontinuous functions (relay, relay with dead zone and saturation, or their combinations), and for various parameters of the membership functions. 


\section{The problem of synthesis and a method for its solution}

The problem of synthesis of fuzzy controllers with approximating nonlinear functions in the control algorithm (CAC-controllers) for the construction of automatic control systems for industrial objects is considered as an integral part of the general process of building and researching the system, including:

- obtaining the dependence of the control signal on the control error using the CACcontroller, which provides the desired mode of operation in the automatic control system at the nominal parameters of the object;

- analysis of the correspondence of the analytical dependence of the approximating characteristic of the control dependence obtained for various membership functions by means of their graphical comparison;

- implementation of the algorithm for correcting the parameters of the original approximating function using fuzzy transformations corresponding to the closest dependence of the controls from those compared.

The static characteristics of the fuzzy controller and the CAC-controller and their corresponding approximating characteristics are given in Table 1, which shows a fairly good correspondence of the approximating control dependencies of systems with different nonlinear characteristics to the control dependencies of the fuzzy controller with different membership functions.

The analysis of the obtained dependences showed (see Table 1) that they can be approximated by a sigmoid function or a combination of sigmoid functions. This makes it possible to implement the following method for the synthesis of a fuzzy controller: to reduce the solution of the formulated problem of synthesis of a fuzzy CAC-controller to the use of the base of rules and membership functions of a known fuzzy controller and an algorithm for a simple correction of the parameters of the CAC-controller, which is solved using known fuzzy transformations based on limited information about the control object and the constraints imposed on the control. This is a new approach to the synthesis of an intelligent system with a fuzzy CAC-controller.

Implementation of a combination of approximating nonlinear functions, in contrast to the work [9], such as «relay + dead zone», «dead zone + saturation», «relay + relay with dead zone + saturation», etc. (Table 1, columns 3 and 5), which lead to obtaining the necessary control dependencies $U(\varepsilon)$ on the control error $\varepsilon$, implemented in a fuzzy controller, provides the required quality of control for different modes. This is confirmed by the obtained quality indicators of transient processes in the automatic control system for various parameters $\lambda, M_{1}$ and $M_{2}$ of the CAC-controller described by the expression:

$$
U(\varepsilon)=\left[\frac{M_{1}}{1+\exp (-\lambda \cdot \varepsilon)}-\frac{M_{1}}{1+\exp (\lambda \cdot \varepsilon)}\right]+\left[\frac{M_{2}}{1+\exp [-\lambda \cdot(\varepsilon-a)]}-\frac{M_{2}}{1+\exp [\lambda \cdot(\varepsilon+a)]}\right],
$$

where $M_{l}, M_{2}$ is the value of the control action in the dead zone (DZ) and beyond, respectively; $\varepsilon$ - the control error; $\lambda$ - the setting parameter; $2 a$ is the size of the dead zone.

The results obtained in the analysis of the influence of parameters $\lambda, M_{1}$ and $M_{2}$ of the CAC-controller (1) on the quality indicators of transient processes in the automatic control system are given in Figure 7. 
Table 1. Types of membership functions for error signals, control action and static characteristics of the fuzzy controller and the CAC-controller.

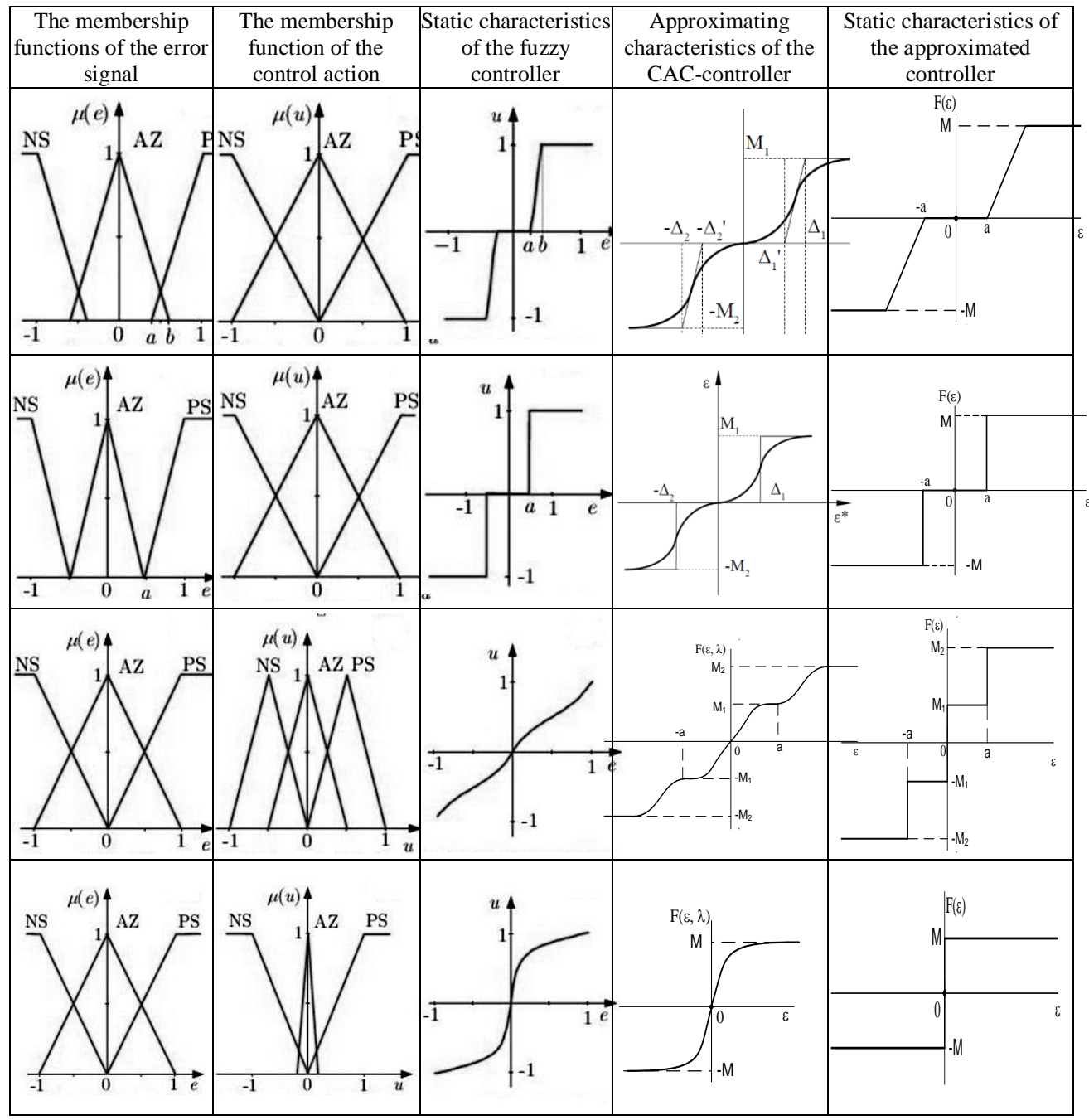




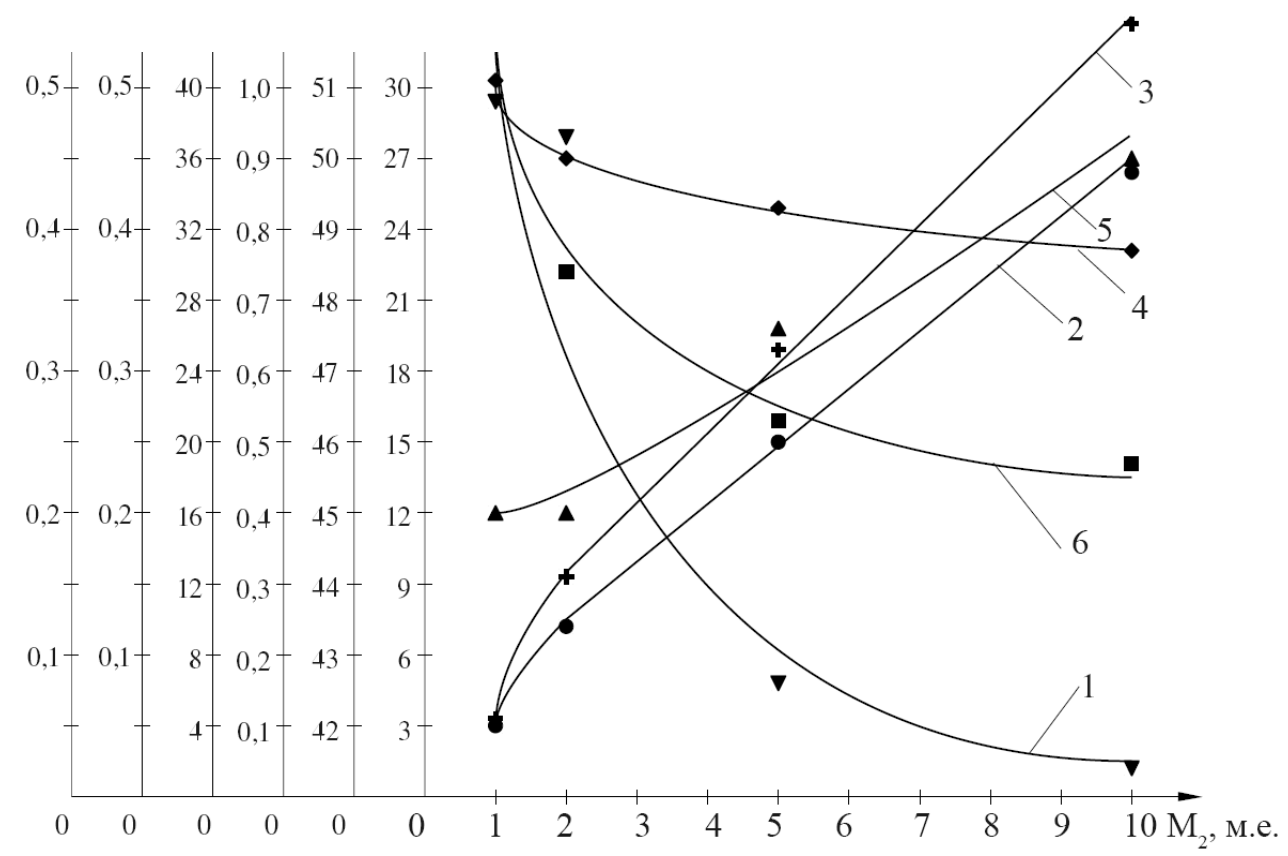

Fig. 7. Dependence of direct quality indicators of control processes in the automatic control system with the CAC-controller at $\lambda=50$ and different values of $M_{2}: 1-$ time of rise $\left(T_{H}\right) ; 2$ - maximum dynamic deviation $\left(A_{\max }\right) ; 3$ - reregulation $(\sigma) ; 4$ - degree of attenuation $(\psi) ; 5$ - time of the transient process $\left(t_{n n}\right) ; 6$ - static error $(\Delta)$.

These results confirm obtaining the desired initial dependences of the approximating control on the mismatch signal, are used when selecting parameters of the membership functions in an algorithm that implements the function of correcting the controller parameters in a fuzzy system.

\section{Results and discussion}

In the course of the research, the following main result with scientific novelty was obtained:

- the method of fuzzy controllers synthesis, which consists in the fact that the rules of fuzzy inference are formed by comparing the control actions of the fuzzy controller and the controller with approximating control;

- membership functions and the rulebase of a known fuzzy controller with the closest control characteristic to the control synthesized on the basis of analytical approximation of the characteristics of typical nonlinear elements are taken as an algorithm for forming the rulebase of a controller with approximating control.

The practical value of the research results is determined by their applicability in solving automatic control problems, namely:

- the use of the controller with approximating control makes it possible to obtain transient processes in multi-mode systems in accordance with the requirements for the main modes: transient and steady-state modes;

- application of the methodology for the formation of a control algorithm using membership functions and the rulebase of the existing fuzzy controller with the closest output characteristic to the control synthesized on the basis of analytical approximation of 
the characteristics of nonlinear elements of the CAC-controller allows eliminating subjective errors in developing the rulebase associated with the human factor;

- the use of a CAC-controllers makes it possible to exclude discontinuous controls, as well as the maximum frequent effects on the actuators, which favorably affects their operation.

\section{Conclusions}

In the course of solving the problem, the expediency of using a combination of typical nonlinear elements in the control algorithms of fuzzy systems of analytical approximation is shown. It is proved that the implementation of fuzzy controllers with control algorithms based on approximating nonlinear differentiable functions does not cause difficulties and does not require significant computational costs. The acceptability of the principle of minimum complexity in the proposed fuzzy system is established: it is sufficient to correct one (out of two) parameters of the fuzzy controller with approximating control for the selected operating mode.

\section{References}

1. Pupkov K A and Egupov N D 2001 Methods of robust, neuro-fuzzy and adaptive control (Moscow: Moscow State Technical University named after N.E. Bauman) $\mathrm{p}$ 744

2. Uskov A A 2004 Instruments and systems. Control, monitoring, diagnostics 6 7-10

3. Pegat A 2009 Fuzzy modeling and control (Moscow: Binom. Laboratory of knowledge) p 798

4. Gostev V I 2011 Design of fuzzy regulators for automatic control systems (St. Petersburg: BHV-Petersburg) p 416

5. Ulyanov S V et al. 2011 Intelligent robust control: soft computing technologies (Moscow: VNIIgeosystem) p 408

6. Uskov A A 2013 Systems with fuzzy models of control objects: Monograph (Smolensk: Smolensk branch of ANO VPO TsS RF «Russian University of Cooperation») p 153

7. Leonenkov A V 2005 Fuzzy modeling in MATLAB and fuzzy TECH (St. Petersburg: BHV-Petersburg) p 724

8. Input-output conversion http://life-prog.ru/1_22017_ preobrazovanie-vhodvihod.html

9. Lubentsov V F 2005 Science and Technology: proceedings of the XXV Russian School on Science and Technology part 2 (Moscow: Russian Academy of Sciences) p 469 